\title{
MODELO ASSISTENCIAL DA CASA DA SAÚDE: REESTRUTURANDO O CUIDADO NA ATENÇÃO PRIMÁRIA NA ITÁLIA
}

\author{
The health care model of "Case della salute": Restructuring primary \\ health care in Italy
}

\section{Ardigò Martino}

Universidade de Bologna-Itália, Professor, Doutor.

Email: ardigma@gmail.com

\section{Brigida Lilia Marta}

Universidade de Bologna-Itália, Medico.

Email: brigida.marta@gmail.com

\section{Francesco Sintoni}

Universidade de Bologna-Itália, Medico.

Email: sintofra@gmail.com

\section{Túlio Batista Franco}

Universidade Federal FluminenseBrasil, Professor Associado, Doutor. Email: tuliofranco@gmail.com

\section{Resumo}

A Casa de Saúde pretende assistir à população nos cuidados primários da clínica integrando ações de prevenção e promoção, bem como atendimentos em algumas especialidades e diagnose. Tem referência territorial e pretende interagir com a comunidade. $\mathrm{O}$ acolhimento e a educação permanente estão presentes como diretrizes. Trata-se de uma pesquisa de caráter exploratório sobre esta recente experiência, em que os aspectos analisados foram aparecendo na medida em que os dados foram sendo produzidos. Como fontes de dados foram utilizados documentos do gestor regional (EmiliaRomagna, Itália) e da Casa da Saúde 'Terre e Fiumi' de Copparo, em Ferrara, além das reflexões resultantes de metodologias qualitativas: observação direta do seu funcionamento, registros de diário de campo, narrativas de gestores, coordenadores de serviços e trabalhadores, atividades de educação permanente. A construção de uma rede de Casas da Saúde é importante para romper com um modelo centrado na atenção hospitalar. Os médicos de família, que antes trabalhavam em seus respectivos consultórios e agora passaram a trabalhar na Casa da Saúde, representam uma transição muito importante, mas no momento insuficiente para caracterizar reestruturação produtiva e trabalho em equipes multiprofissionais, ressaltando-se que os médicos não são funcionários públicos, mas trabalham sob contrato liberal. Também a 
organização dos serviços parece ainda favorecer um modelo de produção do trabalho em saúde fragmentado. Concluise que são necessários investimentos, sobretudo em educação permanente, para promover a reorganização dos processos de trabalho, com o objetivo de proceder a uma reestruturação produtiva, o que pode configurar de fato a mudança do modelo assistencial em um modelo de atenção primária em saúde.

Palavras-chave: atenção primária à saúde; saúde publica; sistemas de saúde.

\section{Abstract}

This paper presents the preliminary findings of an ongoing research concerning a recently introduced innovation in the Italian National Health System, named "Casa della Salute" or CdS (literally, "the house of health"). The research focuses on the experience of the Emilia-Romagna Region (RER). The CdS aims to offer to the population access to the whole health care service, including primary care, health promotion and prevention, as well as outpatient specialist care and diagnostic services. The CdS is responsible for the population of a territory, and is designed to interact with its community. Integrated, person-oriented care strategies as well as continuous education programs - foreseen by the model - are not yet implemented. The data used for the research includes reports from the regional health authorities, reports from the CdS "Terre e Fiumi" ("lands and rivers") in the municipality of Copparo (Ferrara), as well as primary data obtained through qualitative methodologies: direct observation, field notes, narratives collected from key informants (regional health authorities managers and local coordinators of health services) and from permanent education (EP) activities. The results show that the network of services realised through the CdS is a promising step towards moving from the present model of hospital-centred care, and its limits. In order to reach this, the integration of general practitioners into the CdS network will represent a key issue. GPs are traditionally used to work in their offices, while the new model encourages them to move into the CdS. However, this shift seems to be insufficient to promote a real transformation in the model of care. GPs are not public servants and work "outside" of the national health care system. This negatively affects their capacity to work in multidisciplinary teams. In the same way, the current organization tends to maintain a high degree of fragmentation among services. Finally, more investments on the new organizational model are needed, and could be directed particularly towards EP. In our experience, this approach proved to be a powerful tool to foster a reorganization of work processes aimed at transforming the health care system towards a primary health care model.

Keywords: primary health care; public health, health systems

\section{Resumen}

La Casa de la Salud tiene como objetivo ayudar a la población em la clínica de atención primaria de la integración de la prevención y la promoción, así como la atención em algunas especialidades y medios de diagnóstico. Tiene referencia territorial y desea interactuar com la comunidad. El acogimientoy la educaciónpermaniente están presentes como directrices. Se trata de una investigación exploratoria sobre 
esta reciente experiência. Se realizo el análisis mientras que los datos se producen. Como fuentes de datos se utilizaronlos documentos de la Administración Regional de Salud de Emilia-Romagna em Italia, dela Casa de Salud 'Tierra y Ríos' de Copparo Ferrara, además se utilizaron las reflexiones resultantes de los datos recoletadoscon metodologías cualitativas: observación participante, diario de campo, entrevistas narrativas con gerentes, coordinadores de servicios y trabajadores, actividades de educación continua. La construcción de una red de casas de salud es importante para romper con una atención centrada em loshospitales. Algunos de los médicos defamila que antes trabajaban en sus oficinas ahoracomenzaron a trabajaren la Casa de la Salud, esto es una transición muy importante, pero no es todavía suficiente para caracterizar una reestructuración productiva y la producción de trabajo em equipos multidisciplinarios, porque los médicos siguen siendo trabajadores liberales y no funcionarios publicos. Además laorganización estructural de los servicios favorece todavía un modelo de producción em salud fragmentado. Se concluye que es necessario invertir más energía, sobre todo em el aprendizaje permanente, para promover la reorganización de los procesos de trabajo com el fin de llevar a cabo un proceso de reestructuración, lo que puede realmente cambiar el modelo de atención centrado em los hospitales em un modelo de atención primaria ensalud.

Palabras clave: atención primaria de la salud; salud publica; sistemas de salud.
Introdução

Entende-se por transição epidemiológica as mudanças ocorridas no tempo nos padrões de morte, morbidade e invalidez que caracterizam uma população específica e que, em geral, ocorrem em conjunto com outras transformações demográficas, sociais e econômicas. ${ }^{1: 898}$ Este é o processo que se verifica atualmente na Itália, tendo em vista o fenômeno de envelhecimento da população, decorrente, segundo Soliani ${ }^{2}$, de fatores como a taxa de natalidade, fecundidade, morbidade e mortalidade. Na Itália mesmo com variações econômicas, por exemplo, desenvolvimento econômico positivo no período entre 2002 e 2006, ao qual se seguiu uma redução da atividade econômica, mantém-se a tendência de uma população que envelhece, com aumento significativo de anciãos em relação à população economicamente ativa. Importa observar que a transição epidemiológica é uma produção social, que cria interferências no modelo assistencial, que é tensionado para mudar seu padrão de cuidado da população e também os serviços de saúde, na medida em que aumenta a prevalência de problemas de saúde relacionados ao envelhecimento, como as doenças crônico-degenerativas.

A modificação no perfil de saúde da população em que as doenças crônicas e suas complicações são prevalentes resulta em mudanças no padrão de utilização dos serviços de saúde e no aumento de gastos, considerando a necessidade de incorporação tecnológica para o tratamento das mesmas. Tais aspectos representam importantes desafios e fomentam a necessidade de uma agenda para as políticas de saúde que possam dar conta das várias transições em curso.

A questão deste texto vem no sentido de discutir, a partir do processo de transição epidemiológica na Itália, um 


\section{Artigo Original}

novo dispositivo de cuidado que surge no cenário das políticas de saúde que são as "Casas de Saúde", entendendo que tal instância é uma das repercussões do processo vigente, das novas necessidades de cuidado da população, onde a atenção primária é entendida como prioridade.

Primeiramente, apresentam-se alguns dados relativos ao fenômeno do envelhecimento da população italiana, um dos determinantes do processo de transição epidemiológica. Isto justifica a discussão posterior, relacionada ao que se entende ser um processo instituinte, de mudança do modelo assistencial, mesmo que ainda inicial, mas já indica e dá uma ideia do futuro onde a atenção primária tem uma missão importante a cumprir na produção do cuidado. A Itália, assim como outros países com índice de desenvolvimento humano elevado ${ }^{3}$, está experimentando um progressivo envelhecimento da população, os maiores de 65 anos eram $20,3 \%$ do total da população em 2011 , com previsão de ser mais que $32 \%$ a partir de 2043. ${ }^{4}$

Como é possível observar na Figura 1, o envelhecimento da população é acompanhado ainda por uma baixa natalidade, com uma taxa de fecundidade de 1,42 em 2012 (era 1,45 em 2008), com quase 54 mil nascidos(de pais italianos) a menos, em relação àquele ano. Essa tendência é parcialmente compensada com os fluxos migratórios, com a chegada de novos migrantes, entre os quais a taxa de natalidade é mais alta (taxa de fecundidade das mulheres estrangeiras na Itália é de 2,37$).^{5}$

Figura 1 - Pirâmide De População Em 2011 E Perspectivas Para 2065 - Itália.
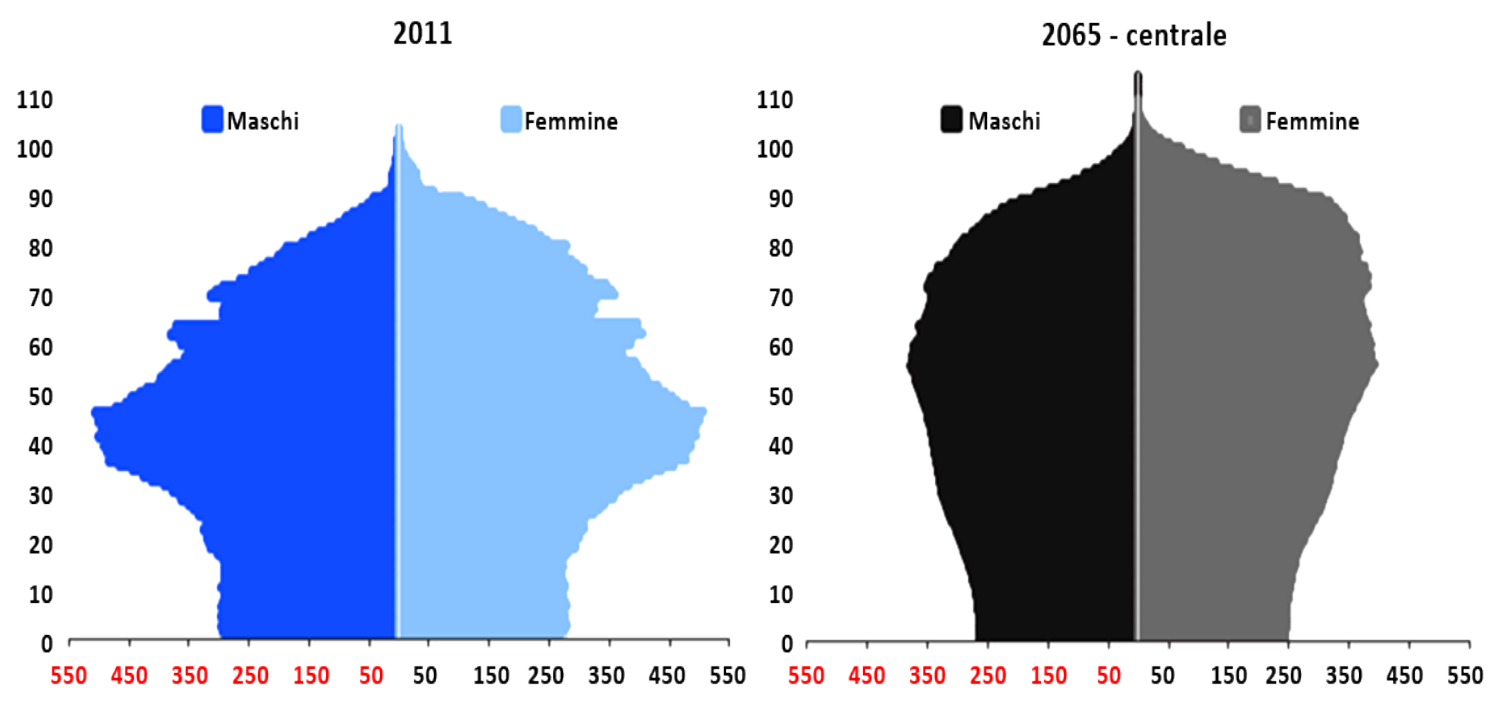

Fonte: Il futuro Demografico del Paese, ISTAT - 28 diciembre de 2011, Itália, p.5.

Estes processos, combinados com as profundas transformações na sociedade devido aos fenômenos ligados à globalização, estão contribuindo para uma rápida transformação do perfil epidemiológico da Itália, com aumento consistente da prevalência das doenças crônicas (segundo as estimativas de 2011 da OMS, 92\% da mortalidade italiana é devida às doenças não comunicáveis) 6 , entre idosos (65-74 anos), 'muito idosos' (a partir de 75 anos) e 'grandes idosos' (como são chamados os maiores de 80 anos), que representam os usuários que mais frequentam e consomem os serviços de saúde na Itália. Esta população em geral apresenta demandas de procedimentos de alta complexidade devido à incidência 
de patologias crônicas graves ou patologias múltiplas, e està aumentando a pressão no sistema de saúde também por conta dos processos ligados à crise econômica (piora das condições gerais de vida e aumento da demanda de saúde, tendo como resposta do sistema as internações hospitalares e consultas especializadas ${ }^{7}$ ). Devido à origem multifatorial das doenças crônicas e as interações complexas entres indivíduos, ambiente e redes sociais, a resposta dos serviços de saúde tem se fundamentado exclusivamente na abordagem biomédica, nos serviços de alta densidade tecnológica (tecnologias duras), especializados e hospitalares. Projetos terapêuticos para cuidados à população em geral, restritos a estes recursos, estão diminuindo a eficácia das intervenções assistenciais, aumentando os custos do sistema, e não respondendo satisfatoriamente às necessidades da população. ${ }^{8}$

Neste sentido tomou-se por referência a ideia de que saúde é um direito fundamental, e cabe aos estados nacionais prover a população serviços e produtos que garantam um cuidado integral. A primeira referência neste sentido vem na Declaração Universal dos Direitos Humanos, adotada e proclamada pela resolução $217 \mathrm{~A}$ (III) da Assembleia Geral das Nações Unidas em 10 de dezembro de 1948.9:3 Acompanhando esta ideia a Constituição da República Italiana adota o princípio de que a saúde é um direito fundamental, garantindo-o no seu Art. 32: "A República tutela a saúde como direito fundamental do indivíduo e interesse da coletividade, e garante o cuidado gratuito aos indigentes." ${ }^{\prime 10}$ Para se responsabilizar pela saúde da população, na dimensão de direito fundamental, é necessário implantar medidas que considerem o perfil epidemiológico, os dados socioambientais e a referência territorial das populações, além de adotar um modelo assistencial centrado na APS e em tecnologias relacionais para a produção do cuidado.
Em 2005, depois da publicação do relatório da OMS sobre doenças crônicas (Preventing chronic diseases: a vital investment ${ }^{11}$ ), o Ministro da Saúde assumiu o compromisso de criar um plano pela prevenção das doenças crônicas, começando com a tradução em italiano e a divulgação do relatório. Logo depois, com a lei n. 296, de 27 de dezembro de 2006 ${ }^{12}$, iniciou-se um processo de implementação de serviços de cuidados primários à saúde, cujo eixo norteador é o modelo da 'Casa da Saúde'. A região Emilia-Romagna está entre as primeiras regiões italianas a adotar o modelo com a lei Dgr 291 do 2010 "Casa della salute: indicazioniregionali per la realizzazione e l'organizzazione funzionale". ${ }^{13}$

O Centro de Saúde Internacional e Intercultural da UniBo, junto a vários colegas de universidades brasileiras (UFRGS, UFF, UFPBA, UFMS) está desenvolvendo, desde 2013, uma pesquisa-intervenção com enfoque em algumas casas da saúde da região, especialmente nas províncias de Bolonha e de Ferrara. Este trabalho apresenta os resultados preliminares da pesquisa.

\section{Materiais e Métodos}

Este artigo é produto de uma pesquisa de caráter exploratório, que tomou como objeto a recente experiência de construção da APS na Itália, região de Emilia-Romagna. Foram produzidos dados através de uma pesquisa quanti-qualitativa ${ }^{14}$ com base na metodologia da pesquisa-formaçãointervenção ${ }^{15}$ participada ${ }^{16,17}$, utilizando-se fontes documentais (dados secundários) produzidas pelo gestor regional quanto à situação epidemiológica na região e às necessidades de saúde da população, assim como relatórios e diretrizes de organização das Casas da Saúde (CS); e também 
documentos produzidos da Casa da Saúde "Terre e Fiumi" localizada em Copparo, município de Ferrara, utilizada como estudo de caso na presente análise.

Esses documentos foram armazenados em uma pasta comum e disponibilizados não somente ao grupo de pesquisa, mas para todos os sujeitos envolvidos e foram utilizados como materiais para as atividades de educação permanente e para as atividades de análise participativa das informações.

Juntamente com tal base empírica, foram utilizados como instrumentos para reflexão e elaboração do presente relato e utilizando metodologias etnográficas ${ }^{18}$ : diário de campo individual e coletivo, entrevistas com informantes chaves, entrevistas biográficas e narrativas, e ferramentas de análise baseadas no processo de educação permanente $\mathrm{e}^{19,20: 247} \mathrm{e}$ de produção de cuidado em saúde ${ }^{21}$, além da análise textual dos relatórios das oficinas realizadas com gestores, profissionais e comunidade. A observação deu-se tomando por referência os aspectos de estrutura, funcionamento e resultados esperados com a sua organização. Na oportunidade foram tomadas narrativas dos gestores e coordenadores de serviços das CS, e dos profissionais dos serviços que relataram os aspectos funcionais e os desafios das mudanças que estão sendo programadas, sendo conjuntamente analisados os posicionamentos e as relações entre os diferentes atores.

$\mathrm{O}$ que se aborda neste artigo referese às atividades realizadas entre janeiro de 2013 e maio de 2015. A partir destas atividades foi realizada uma triangulação das informações ${ }^{22}$ obtidas por diferentes fontes informativas e metodologias. Os diários de campo, as transcrições das entrevistas e a descrição do processo de organização em curso foram analisados mediante metodologias de análise textual, análise discursiva e análise conceitual. ${ }^{23}$
Foram analisados de forma participativa os conteúdos éticos, simbólicos e a forma como o processo de implantação da Casa da Saúde é apresentado e justificado. O objetivo pactuado dessa primeira fase foi definir o contexto material e simbólico onde a experimentação está se desenvolvendo, os princípios declarados e não declarados, e o posicionamento dos atores envolvidos. Essas informações são consideradas relevantes na avaliação da pesquisa que está sendo realizada, além da produção de trabalho em saúde realizada nas Casas da Saúde em estudo. A tentativa é de realizar os ajustes necessários para as próximas etapas da pesquisa intervenção e dar sugestões aos gestores e profissionais envolvidos quanto à revisão da arquitetura do sistema e da produção do trabalho.

\section{Resultados e Discussão}

No período relatado foram promovidas cerca de 200 horas de atividades de educação permanente com trabalhadores dos serviços sociais, profissionais da Casa da Saúde, médicos de medicina da família e gestores. Foram realizadas 36 visitas técnicas em quatro Casas da Saúde e cinco atividades de extensão em duas Casas da Saúde envolvendo estudantes da UniBo, profissionais e gestores do serviço de saúde e social. Duas atividades foram realizadas articulando os profissionais da Casa da Saúde e a comunidade, e 16 encontros técnicos com três núcleos de trabalho que estão experimentando a implantação de atividades similares ao do Agente de Saúde, mas dirigidas à população vulnerável. Foram efetivados outros 75 encontros técnicos com gestores de serviço e gestores regionais do sistema de saúde da região Emilia-Romagna e 20 encontros com responsáveis do Curso de Enfermagem e Medicina, na UniBo, para a discussão quanto à realização de atividades 
de extensão como estratégias para adaptar o currículo ao novo cenário epidemiológico e de organização dos serviços. Quanto à análise documental, foram coletadas as leis que fundamentam a constituição da Casa da Saúde na Itália e na região da Emilia-Romagna, os relatórios técnicos e os documentos internos do sistema de saúde.

\section{A Construção da Atenção Primária à Saúde na Região Emilia-Romagna e a produção de cuidado}

Dos temas levantados na pesquisaação, são aqui desenvolvidos dois: a dificuldade de mudar o sistema de valores do serviço, centrado na doença, para focar na saúde dos sujeitos; e a permanência de tensões políticas, econômicas, sociais e culturais que estão produzindo fragmentação e falta de coerência no campo das ações de cuidado em saúde.

Inicialmente, pode-se afirmar sem muita surpresa que a Casa da Saúde representa uma novidade relativa no sistema de saúde, nem todos os usuários e profissionais estão cientes da existência desse dispositivo de cuidado, mesmo ela estando implantada na Região de EmiliaRomagna e em outras regiões. Trata-se de uma unidade de saúde próxima do que no Brasil se conhece por Unidade Básica do Sistema Único de Saúde (SUS). O aspecto mais importante é o fato de que até então os serviços de assistência à saúde territoriais, na Itália, estiveram sempre organizados de forma fragmentada e com uma logica operacional referente aquela hospitalar, mas desde 2009, mediante uma proposta do governo nacional de adesão voluntária de experimentação dirigida às Regiões (Presidente do conselho Romano Prodi, de centro esquerda), algumas Regiões começaram a organizar novos serviços de APS. A DGR no 427 de 2009 'Diretrizes para implementação regional do PPSR 20082010 para a qualificação dos cuidados primários através do desenvolvimento dos profissionais de assistência à saúde' identifica novos modelos organizativos e assistenciais que possam ser tomados como referência para ofertar uma assistência coerente com a necessidade de saúde, e enquanto apresenta uma importante oportunidade para avaliar e validar novos papéis e responsabilidades profissionais.

A Região Emilia-Romagna é uma região historicamente ligada à esquerda política italiana, portanto pode-se ressaltar que a adesão da administração regional ao modelo proposto pelo governo central de centro-esquerda manifesta conotação política, além de técnica. Esse ponto é importante porque configura uma mudança de sistema produzida de forma top-down, em um contexto onde o nível de reflexão da comunidade, de usuários, profissionais e gestores não é ainda suficiente para suportar uma mudança tão radical. Outro elemento importante é que tal opção coincide com a recente crise econômica na região do euro, resultando em menor capacidade fiscal dos governos em financiar os caros serviços de saúde. Fechar os hospitais torna-se assim mais fácil para os políticos, podendo mostrar à população que está em processo a constituição de uma alternativa apresentada como mais eficaz e mais eficiente. Da mesma forma não surpreende uma forte resistência a esse processo expressa quer pela minoria política no parlamento regional e nas prefeituras, por vários profissionais e gestores, e mesmo por uma parte consistente da comunidade, que em algum contexto chegou a ocupar fisicamente hospitais para impedir que fossem fechados. Se de um lado a motivação governamental vai ao encontro da organização de serviços de atenção primária, pela necessidade política e pelas vantagens de custos que estes representam, por outro se pode constatar que se abre uma oportunidade única de vivenciar um novo modo de funcionamento dos serviços. 
Novas referências de cuidado colocam-se na cena de discussão das políticas de saúde, do processo de trabalho dos médicos e demais profissionais, que ganham centralidade na agenda dos gestores de serviços. Além do mais, a relação com os usuários passa a ocupar um campo próprio e importante de discussões entre aqueles pensam esta nova modalidade assistencial.

De acordo com o Relatório da Região de Emilia-Romagna publicado em $2011^{24: 7}$, iniciou-se a organização junto aos Departamentos de Atenção Primária, e suas articulações territoriais: os Núcleos de Cuidados Primários (NCP), cuja localização constitui um dos elementos fundamentais para favorecer o melhor cuidado dos usuários. Trabalha-se aqui a integração dos profissionais, prioritariamente os médicos de medicina geral, médicos de continuidade assistencial, e pediatras com liberdade de escolha do usuário, os especialistas ambulatoriais, os enfermeiros, obstetras e assistentes sociais. A evolução dos NCP e sua inserção na rede de serviços e saúde, assim como a sua constituição como referência territorial para os usuários, conduziu ao que se constituiu nas Casas da Saúde (CS).

A missão da Casa da Saúde está definida da seguinte forma:

A realização das CS está inserida no percurso de redefinição dos serviços territoriais iniciados na Região da Emilia-Romagna para melhorar o acolhimento, a proximidade do cuidado e a continuidade assistencial. As CS começaram pela Agência de Inovação para a Assistência Primária, tendo como objetivo a consolidação de iniciativa estrutural e organizativa segundo um modelo multidisciplinar e interprofissional com esclarecimento do papel e da relação dos e entre os atores. As CS são estruturas de saúde e sócio-sanitárias do núcleo de cuidados primários com o objetivo de fornecer aos cidadãos um ponto de referência certo, distribuído de modo homogêneo ao qual é possível recorrer em cada hora do dia. A CS "Terre e Fiumi" identifica como sua finalidade e coloca no centro do próprio modelo (visão) a resposta às necessidades de saúde buscando a tempestividade, a essencialidade e a adequação, a continuidade dos cuidados e a integração com os outros profissionais, a fim de garantir a produção e prestação dos serviços essenciais em relação às necessidades dos cidadãos." ${ }^{25}$

Nesse texto, encontram-se as principais diretrizes que norteiam a ideia de organização de 'Casas da Saúde' da região de Emilia-Romagna na Itália.

Em articulação com os princípios gerais do que foram as ideias originais da APS, o modelo italiano é herdeiro de sua própria história, combina princípios que vêm da construção da atenção primária desde a Conferência de Alma-Ata, com a especificidade da realidade local, por exemplo, o fato de considerar a clínica e o cuidado como componentes e associados às diretrizes com base na vigilância à saúde e no território. É possível perceber o interesse na constituição de um serviço que opere a clínica já instituindo a ideia de inter e multidisciplinaridade, e ao mesmo tempo processos de prevenção e promoção à saúde. Em relação aos seus objetivos o documento de apresentação da proposta diz o seguinte: "Os objetivos gerais têm por referência as indicações e instruções das diretrizes regionais, e são atribuíveis aos seguintes itens: assegurar um único ponto de acesso ao cidadão; garantir continuidade da assistência 24 horas nos 7 dias da semana; organizar e coordenar a resposta ao cidadão; reforçar a integração com o hospital com particular respeito à proteção, cuidado integral de algumas patologias crônicas, compartilhamento de diretrizes e protocolos assistenciais para patologias que requerem recursos de elevada complexidade; melhorar o cuidado integral 


\section{Artigo Original}

à pessoa com problemas de saúde mental; desenvolver programas de prevenção dirigidas ao indivíduo; à comunidade, a alvos específicos; promover integração com serviços sociais e com a rede de serviços sócio-sanitários; promover e valorizar a participação dos cidadãos; oferecer educação permanente aos trabalhadores." Através das atividades de pesquisa é possível apreender que há muito o que fazer do ponto de vista prático e operacional, sobretudo para a constituição de equipes, do trabalho multiprofissional e da produção de um cuidado integral.

Mais pontualmente pode-se ressaltar que um primeiro limite é representado pela organização do serviço e um segundo pela formação dos profissionais, pois se percebe muito claramente o peso da história do sistema e as dificuldades em se adaptar às mudanças. Do ponto de vista organizacional, os objetivos gerais da Casa da Saúde vão ao encontro de um tipo de funcionamento de atenção às necessidades dos usuários, olhando inclusive aqueles que trabalham. As Casas de Saúde mantêm a oferta de serviços também à noite $(24 \mathrm{~h}$, por 7 dias da semana) propondo-se a funcionar, inclusive, como retaguarda do hospital, recebendo egressos de internação, garantindo a continuidade dos cuidados e combinando programas de prevenção, integrando essas atividades com a comunidade, valorizando a participação dos usuários.

Na prática o processo de empresariamento (aziendalizzazione), iniciado em 1992, com a lei $502^{26}$ tem produzido a entrada da lógica do gerenciamento econômico no sistema de saúde italiano. Com o decreto legislativo n. 229 de 19 de junho de 1999 a responsabilidade do serviço de saúde passa às Regiões, as quais herdam a lógica de organização e avaliação de tipo empresarial. A unidade organizacional do serviço de saúde italiano é a AUSL' ('Azienda' Unidade de Saúde Local). A AUSL tem responsabilidade de oferta de serviços de saúde, gestão, avaliação dos serviços e vigilância sanitária e epidemiológica. Nessa estrutura fundamental do sistema, os diferentes serviços são organizados de forma verticalizada, como evidenciado pela Figura $2^{27}$, que mostra o organograma da AUSL de Modena.

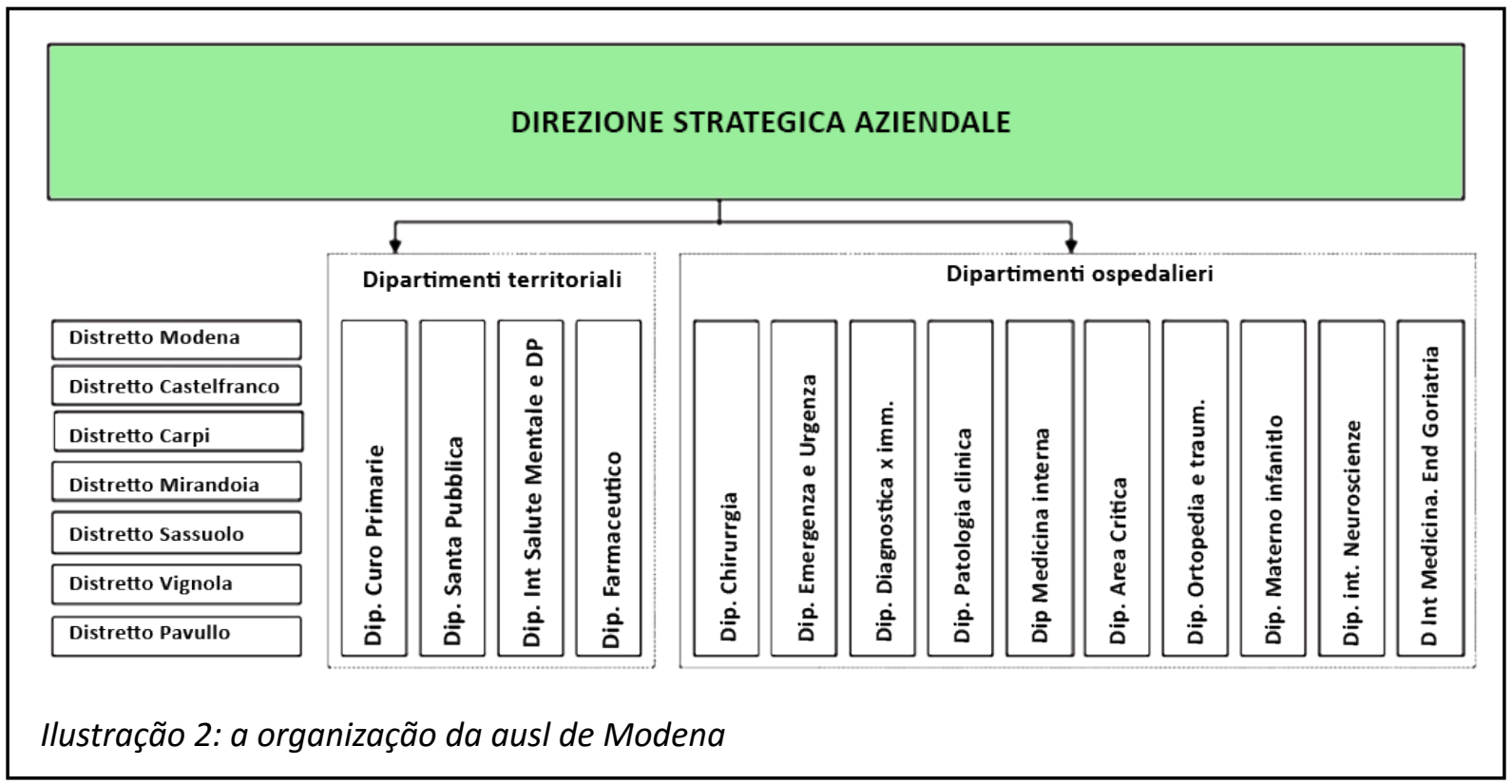

\footnotetext{
- O AUSL é uma entidade pública da República Italiana. Cada uma das 21 Regiões da República é dividida em várias AUSL, que são empresas com personalidade jurídica pública e são centros de imputação de autonomia empresarial. O AUSL tem autonomia organizacional, gerencial, técnica, administrativa, financeira e contábil. Eles devem cumprir os deveres do sistema nacional de saúde italiano em uma determinada área geográfica. Em termos concretos, são as Autoridades de Saúde Pública local, são os responsáveis por os cuidados de saúde para a população de um território e são produtores e/ou compradores de serviços.
} 
Serviços, mas constitutivamente separa os diferentes "pedaços" da atenção que conformam o cuidado. Além da separação entre serviços territoriais e serviços hospitalares, chama à atenção a separação entre o departamento de Cuidado Primário e o departamento de Saúde Pública, duas funções fundamentais na atenção primária e na produção de integralidade. Além disso, como acontece em muitos outros países, o sistema de serviços sociais é separado da saúde, produzindo ainda mais fragmentação, especialmente em relação aos doentes crônicos, idosos, de baixa renda e/ou com outros problemas sociais relevantes. A última consideração é a respeito da integração território-serviço de APS e entre serviço de APS com os níveis superiores. Do lado da integração territórioserviço, é visível a falta de um profissional com funções parecidas ao agente de saúde. Sendo assim, a Casa da Saúde não muda realmente seu papel de sistema curativo a sistema preventivo-promotor-curativo. ${ }^{28: 44}$ Por outro lado, na presente experiência, nas Casas da Saúde onde se organizam atividades de encontro com a comunidade, registrou-se uma dificuldade na realização desses encontros. Os profissionais e a "comunidade" não têm uma ideia clara de como esse encontro deveria acontecer e nas reuniões presenciadas, registrouse uma dificuldade de comunicação e de pactuação. Desse modo, mesmo declarado como objetivo torna-se muito difícil realizar a integração entre sistema primário e outros níveis, dificultando também a produção de um sistema de cuidado integral e a realização de um sistema de referência e contra referência eficaz. ${ }^{29: 549}$

Outra dificuldade relevante é representada por uma anomalia do sistema de saúde italiano, ou seja, a forma de contratualização do médico de família. No modelo italiano, os médicos colocam-se "fora" do sistema de saúde, são remunerados por captação e trabalham em seus consultórios privados com base em um contrato com o sistema público de saúde. A adesão ao modelo da Casa da Saúde é, até o momento, feita de forma voluntária e o sistema não tem como forçar esses profissionais médicos a aderir nem tem instrumentos para modificar os processos de produção de trabalho. Sendo assim, os médicos de família podem se recusar a trabalhar na Casa da Saúde, e mesmo quando da transferência física para dentro da estrutura, podem se recusar a trabalhar em equipe com os outros profissionais que, pelo contrário, são contratualizados pelo Serviço Nacional de Saúde. Outra questão fundamental referese à distribuição de médicos de família na população. O médico de família é escolhido por usuários que moram na mesma cidade, independentemente de adscrição territorial. Portanto, pacientes que são atendidos por um médico de família podem pertencer a uma Casa da Saúde diferente daquela a que o médico de família serve. Nesse cenário, sem a adequação e os ajustes necessários, a assistência do médico pode se realizar de forma totalmente 'aterritorializada', com uma produção de clínica muito voltada à intervenção do modelo biomédico, mas pouco ao modelo de atenção primária.

Na verdade, de acordo com o próprio Relatório da Região Emilia-Romagna, o médico trabalhando isolado no seu consultório funciona mais como uma porta de entrada para o sistema, e há uma carga burocrática de trabalho muito grande, reduzindo seu trabalho assistencial. Por outro lado, o trabalho na CS

[...] restitui ao Médico de Medicina Geral o papel clínico, e também a titularidade da função de governo sobre o trabalho na clínica, valorizando, a contento, os enfermeiros na atividade de cuidado, aconselhamento, assistência, acompanhamento no itinerário terapêutico, adesão à terapia." $24: 10$ 
A mudança no processo de trabalho na situação estudada ainda é insuficiente para caracterizar uma mudança no modo de produzir o cuidado.

A alteração da contratualidade do médico é uma questão importante, pois, na sua quase totalidade mantém um contrato como profissional liberal, colocando-se separado do sistema de saúde, e desta forma, com baixa possibilidade de: constituição de vínculo necessário, responsabilização, acompanhamento dos projetos terapêuticos dos usuários e especialmente constituição de rede com os demais profissionais da saúde. Os que têm aderido à proposta de CS declaram inicialmente o apoio à experiência, e consideram melhora no seu processo de trabalho. Mas a própria experiência da CS é inicial e esta questão merece uma atenção especial pela centralidade que tem no desenvolvimento do modelo assistencial e o modo de produção do cuidado.

A associação da clínica com as atividades de prevenção de doenças na Casa da Saúde se dá, por um lado, pela natural necessidade de cuidados clínicos que se verifica nas redes de atenção primária. Esta associação da clínica e o campo da vigilância à saúde é importante para a integralidade do cuidado. Isto não significa que havia um processo de reestruturação produtiva até então, ou seja, que as práticas biomédicas tenham sido substituídas por outros modelos e processos de trabalho para o cuidado à saúde. Com base em observações mais próximas ao cotidiano dos serviços e sua micropolítica deverá ser avaliado se há um processo de inversão das tecnologias de trabalho nos processos produtivos, por exemplo, com prioridade das tecnologias leves e o uso das tecnologias duras e leve-duras na medida exata da sua necessidade. ${ }^{30: 180}$

Do ponto de vista das diretrizes assistenciais formuladas para a Casa da Saúde há uma intenção declarada de produzir um processo de reestruturação produtiva, ou seja, não apenas alterar as referências de rede de cuidado à saúde, deslocando a centralidade do hospital para uma rede da APS, mas construir um novo modo de produzir o cuidado, como se pode ver abaixo:

Os aspectos e princípios da Casa da Saúde são o acolhimento e a orientação nos serviços, sócio-sanitários e assistenciais, a assistência sanitária para problemas ambulatoriais urgentes, a gestão de patologias crônicas, através da integração da assistência primária com serviços especializados, os itinerários para diagnósticos e as intervenções de prevenção e promoção da saúde. ${ }^{24}$

Torna-se especialmente interessante o fato de que a ideia do acolhimento está presente nas diretrizes, tratando-se de dar um conteúdo à ela, atribuindo ao conceito o que propõe Franco, Bueno e Merhy: como um atender bem, encaminhar, resolver, se responsabilizar, e para isto é necessário mudar os processos de trabalho. Esta mudança deve vir na direção de uma maior integração no trabalho dos profissionais, compondo de fato a interdisciplinaridade, um processo de trabalho em rede, centrado no usuário e uso central das tecnologias leves. É importante a instituição desta diretriz, que cria a possibilidade de um processo de reestruturação produtiva de fato, que possa inverter o modelo de produção do cuidado.

Através das atividades de pesquisa tem-se evidenciado uma dificuldade dos profissionais em transformar os hábitos de trabalho, sendo três os maiores temas relacionados com a mudança de trabalho: a formação universitária; a história consolidada de produção de trabalho em saúde dos profissionais e do serviço; e a dificuldade dos gestores em aceitar mudanças na produção de trabalho sem poder ter indicadores de resultado, sendo 
que eles são vinculados à produção de prestações com metas. É importante registrar que os profissionais envolvidos na pesquisa têm valorizado muito a perspectiva da educação permanente como uma ferramenta fundamental na constituição dos serviços. Tal possibilidade se conecta com a necessária manutenção de programas formativos, servindo os mesmos como dispositivo para qualificação dos serviços e mudanças no processo de trabalho.

A respeito da formação existem vários elementos levantados. Os profissionais com experiência maior lamentam a falta de formação para trabalhar no novo cenário. Quando a formação é oferecida, nem sempre é considerada adequada ao contexto de trabalho do profissional, mesmo sendo avaliada como de boa qualidade. Quando a formação de boa qualidade é compatível com o serviço, outra questão que emerge é a dificuldade dos gestores em conceder mudança na organização do trabalho. Profissionais lamentam que o gestor não os deixem colocar em ato as boas práticas que eles têm aprendido ao longo do curso. Por último, tanto os gestores quanto os jovens trabalhadores lamentam a distância entre a formação acadêmica e as competências necessárias para trabalhar no novo cenário. $A$ respeito disso, a distância entre universidade e mundo do trabalho é considerado um problema pelos profissionais, pelos gestores e também pelos políticos. Partindo do pressuposto que é necessário gerar uma reestruturação produtiva para que a Casa da Saúde cumpra o que está definido nas suas diretrizes, entende-se que este processo deva ter a educação permanente como dispositivo para fomentar mudanças necessárias, operando principalmente sobre a micropolítica do processo de trabalho.

A educação permanente consiste em um processo de educação centrado no trabalho, tomando os cenários de práticas de cuidado e todas as atividades do cotidiano como insumos para o processo de ensino- aprendizagem do profissional de saúde. ${ }^{31}$ Por exemplo, se uma equipe que cuida de um usuário vem empreendendo uma série de atividades no seu projeto terapêutico, este pode servir como elemento para o processo de educação. Busca-se então analisá-lo com base nas narrativas dos próprios profissionais, remontando a história do usuário e todo seu percurso no serviço de saúde, em detalhes, tendo como foco o trabalho e as relações entre os próprios profissionais e destes com os usuários. Tal estratégia possibilita uma análise detalhada do processo de trabalho e do modo como se produz o cuidado, e como são os profissionais que fazem a análise; sendo eles protagonistas deste trabalho, a análise se volta para eles mesmos, operando assim um processo também de autoanálise. A autoanálise é a possibilidade de crítica sobre seu próprio processo de trabalho, abrindo para uma ruptura com aquilo que ele mesmo considera inadequado, e para a construção de novas práticas e relações no ambiente de cuidado.

Partindo da educação permanente tal como pensada aqui, consideram-se as unidades de produção do cuidado como unidades de produção pedagógica. ${ }^{32}$ Sendo assim toda atividade de trabalho na Casa da Saúde pode ser atividade de ensinoaprendizagem. E os processos em curso podem operar este processo de educação em duas dimensões: a cognição, que se refere ao conhecimento técnico; e a subjetivação, que diz respeito à mudança das subjetividades. Enfim é necessário investir na mudança da micropolítica do processo de trabalho para que ocorra uma mudança no modelo de produção do cuidado. Esta é uma possibilidade já experimentada em outros serviços e, portanto com demonstração do grande potencial da educação permanente como dispositivo de mudança e qualificação da atenção primária. ${ }^{33}$ 


\section{Considerações Finais}

A experiência verificada até aqui indica um importante progresso em relação ao modelo anterior, em que a assistência à saúde estava centrada no trabalho de tipo hospitalar, que era utilizado para todo tipo de cuidado, e não apenas àquele que necessitava de uma tecnologia hospitalar específica. A "Casa da Saúde" é uma experiência recente que traz elementos positivos no sentido de compor uma integralidade dos serviços, associando a clínica e ações de prevenção e promoção, com inserção na sociedade e participação dos usuários. É ainda uma experiência limitada territorialmente, pois está reduzida a apenas algumas regiões italianas. Nos lugares em que está implantada é possível disparar um processo de reestruturação produtiva, ou seja, o deslocamento de grande parte das práticas de cuidado hospitalares para este equipamento, configurando um novo tipo de cuidado à saúde, com base em um outro processo de trabalho.
O processo é inicial e, portanto, há muitos elementos e serem trabalhados, como por exemplo, o que se refere à composição de equipes multiprofissionais, à qualificação das relações entre profissionais e usuários, onde os espaços de fala e escuta entre ambos seja valorizado como prática cotidiana do cuidado e da clínica. Mais do que as Casas da Saúde que fortalecem a atenção primária, é necessário operar mudança nos processos de trabalho, uma questão fundamental para o sucesso desta experiência, naquilo que ela se propõe, conforme declarado em seus documentos.

No momento o mais importante é acompanhar de perto a experiência, procurando interagir com os processos em curso, e na medida do possível compor com os trabalhadores e usuários que estão envolvidos no esforço de mudar o modelo de assistência à saúde na Região de Emilia-Romagna na Itália. Ao mesmo tempo considera-se fundamental disparar processos de análise, educação e aprendizado da experiência, usando como proposta a educação permanente.

\section{Referências}

1. Schramm JMA et al. Ciência \& Saúde Coletiva, 2004; 9(4):897-908.

2. Soliani L. La popolazione: previsionidemografiche 2010-2030. Disponível em: <http://www. ilborgodiparma.it/documenti/Parma_Citta_Comunita/LA\%2OPOPOLAZIONE1.pdf>. Acesso em: ago 2014.

3. HumanDevelopment Index. Disponível em: <http://hdr.undp.org/en/statistics/hdi>. Acesso em: jul 2014.

${ }^{4}$. Il futuro demograficodelpaese, 2011. Disponível em: <http://www.istat.it/it/archivio/48875>. Acesso em: jul 2014.

5. Natalità e feconditàdellapopolazione residente, 2013. Disponível em: <http://www.istat.it/ it/archivio/104818>. Acesso em: jul 2014.

${ }^{6}$. World Health Organization - NCD Country Profiles , 2011. Disponível em: <http://www.who. int/nmh/countries/en/>. Acesso em: jul 2014. 


\section{Artigo Original}

7. STAT (a cura di) La salute e ilricorsoaiservizisanitariattraversolacrisi 2013. Disponível em: <http://www.istat.it/it/archivio/108565>. Acesso em: jul 2014.

${ }^{8}$. Progetto Presidio Casa dellaSalute( CdS ) Regione Toscana Adeguamentoedimplementazione.

9. Organização das Nações Unidas. Declaração Universal dos Direitos Humanos. UNIC / Rio / 005 - Dezembro 2000.

${ }^{10}$. Repubblica Italiana. Constituzzione de laRepubblica. Disponível em: <http://www.governo. it/Governo/Costituzione/principi.html>. Acesso em: jul 2014.

${ }^{11}$. Tunstall-Pedoe H. "Preventing Chronic Diseases. A Vital Investment: WHO Global Report. Geneva: World Health Organization, 2005; p. 200. CHF 30.00. Also publishedon http://www. who.int/chp/chronic_disease_report/en." International Journalof Epidemiology 35.4 (2006): 1107-1107.

12. Italia. Presidencia della Repubblica. Legge n. 296, de 27 de dezembro de 2006. Disponível em: <http://www.normattiva.it/uri-res/N2Ls?urn:nir:stato:legge:2006-12-27;296>. Acesso em: jul 2014.

13. Italia. Giunta della regione Emilia Romagna. Progr.Num. 291/2010. Disponível em: <http://salute.regione.emilia-romagna.it/documentazione/leggi/regionali/delibere/dgr291-2010-201ccasa-della-salute-indicazioni-regionali-per-la-realizzazione-e-lorganizzazionefunzionale201d/at_download/file/Dgr.\%20291.2010.pdf>. Acesso em: jul 2014.

${ }^{14}$. Pope C, Mays N, Popay J. Synthesising Qualitative and Q uantitative Health Evidence: A Guide to Methods: A Guide to Methods. McGraw-Hill Education (UK), 2007.

15. Ceccim RB, Feuerwerker LCM. O quadrilátero da formação para a área da saúde: ensino, gestão, atenção e controle social. Physis, 2004; 14(1): 41-65.

16. Baum F, MacDougall C, Smith D. Participatory action research.Journal of epidemiology and community health, 2006; 60(10): 854.

17. McIntyre A. Participatory action research, 52. Sage Publications, 2007.

${ }^{18}$. Silverman D. Qualitativeresearch. Sage, 2010.

19. Ceccim, RB. Educação permanente em saúde: desafio ambicioso e necessário. Interface Comunic , Saúde, Educ., 2005; 16(9):161-168.

20. Haddad JQ, Roschke MAC, Davini MC. Educacion permanente de personal de salud. Washington, D.C.: OPS, 1994.

${ }^{21}$. Franco TB, Merhy EE. A produção imaginária da demanda e o processo de trabalho em saúde. Construção social da demanda: direito à saúde, trabalho em equipe, participação e espaços públicos. Rio de Janeiro: Cepesc/Uerj/Abrasco, 2005:181-193.

22. Denzin, Norman K. “Triangulation 2.0." Journal of Mixed Methods Research 6.2 (2012): 8

${ }^{23}$. Silverman, David, ed. Qualitative research. Sage, 2010.

${ }^{24}$. Regione Emilia-Romagna, Direzione Generale Sanitá e Politiche sociali "Le Case della Salute funzionanti, La rete dei Professionisti." 


\section{Artigo Original}

${ }^{25}$. Documento dipresentazionedella Casa dellaSalute. Terre e Fiumi Asl Ferrara, RegioneEmiliaRomagna

${ }^{26}$. Ferré F et al. Italy: Health system review. 2014.

27. http://www.ausl.mo.it/flex/cm/pages/ServeBLOB.php/L/IT/IDPagina/8447

${ }^{28}$. Mendes EV. O cuidado das condições crônicas na atenção primária à saúde: o imperativo da consolidação da estratégia da saúde da família. O cuidado das condições crônicas na atenção primária à saúde: o imperativo da consolidação da estratégia da saúde da família. Organização Pan-Americana da Saúde, 2012. 54

29. Mendes, Eugênio Vilaça. "As redes de atenção à saúde."Brasília: Organização Pan-Americana da Saúde (2011): 549.

30. Merhy EE. Saúde: Cartografia do Trabalho Vivo. São Paulo: Hucitec; 2002.

31. Franco TB, Bueno WS, Merhy EE. O Acolhimento e os Processos de Trabalho em Saúde: o caso de Betim (MG). Cadernos de Saúde Pública. 1999; 15(2): 345-353.

32. Franco TB. Health care production and pedagogical output: integration of health care system settings in Brazil. Interface (Botucatu) [online]. 2007; 11(23): 427-438.

33. Pinto S et al. Tecendo Redes: Os planos da educação, cuidado e gestão na construção do SUS. São Paulo: Hucitec; 2012. 